Full length article

\title{
Collagenase nanocapsules: An approach to fibrosis treatment
}

\author{
M. Rocío Villegas ${ }^{\mathrm{a}, \mathrm{b}}$, Alejandro Baeza ${ }^{\mathrm{a}, \mathrm{b}, *}$, Alicia Usategui ${ }^{\mathrm{c}}$, Pablo L Ortiz-Romero ${ }^{\mathrm{d}}$, José L. Pablos ${ }^{\mathrm{c}}$, \\ María Vallet-Regí ${ }^{\mathrm{a}, \mathrm{b}, *}$
}

a Departamento de Química en Ciencias Farmaceúticas, Facultad de Farmacia, Universidad Complutense de Madrid, 28040 Madrid, Spain

${ }^{\mathrm{b}}$ Networking Research Center on Bioengineering, Biomaterials and Nanomedicine (CIBER-BBN), Spain

' Servicio de Reumatología, Instituto de Investigación Hospital 12 de Octubre (I+12 Medical School), Universidad Complutense de Madrid, Spain

${ }^{\mathrm{d}}$ Servicio de Dermatología, Instituto de Investigación Hospital 12 de Octubre (I+12 Medical School), Universidad Complutense de Madrid, Spain

\section{A R T I C L E I N F O}

\section{Article history:}

Received 5 February 2018

Received in revised form 19 April 2018

Accepted 3 May 2018

Available online $\mathrm{xxxx}$

\section{Keywords:}

Fibrosis

Collagenase

Polymeric nanocapsules

Enzymatic injection

\begin{abstract}
A B S T R A C T
Fibrosis is a common lesion in different pathologic diseases and defined by the excessive accumulation of collagen. Different approaches have been used to treat different conditions characterized by fibrosis. The FDA and EMA approved the use of collagenase to treat palmar fibromatosis (Dupuytren's contracture). The EMA approved additionally its use in severe Peyronie's disease, but it has been used off label in other conditions $[1,2]$. The approved treatment includes up to three (in palmar fibromatosis) or up to eight (in penile fibromatosis) injections followed by finger extension or penile modeling procedures, typically causing severe pain. Frequent single injections are adequate to treat palmar fibromatosis [3]. The need to repeatedly inject doses of this enzyme can be due to the labile nature of collagenase, which exhibits a complete activity loss after a short period of time. This study presents a novel strategy to manage this enzyme based on the synthesis of polymeric nanocapsules that contain collagenase encapsulated within their matrix. These nanocapsules have been engineered for achieving a gradual release of the encapsulated enzyme for a longer time, which can be up to ten days. The efficacy of these nanocapsules has been tested in a murine model of local dermal fibrosis, and the results demonstrate a reduction in fibrosis greater than that with the injection of free enzyme; this type of treatment showed a significant improvement compared to conventional therapy of free collagenase.
\end{abstract}

\section{Statement of Significance}

The use of proteins as therapeutic molecules has recently attracted great interest. Collagenase injection is the current treatment for fibrotic diseases. Unfortunately, proteins have a low stability and presume several repetition cycles to obtain an effective treatment. This article describes a novel treatment for these types of diseases using collagenase nanocapsules designed to exhibit a sustainable release of the encapsulated enzyme, which maintains the enzymatic activity for a long period of time. The therapeutic effect of nanocapsules was tested in a murine mouse model of local dermal fibrosis, and the results showed an important improved effect compared to the effect of the administration of free enzyme. These results indicate a high potential for this novel system to improve the current treatment for fibrotic diseases.

() 2018 Acta Materialia Inc. Published by Elsevier Ltd. All rights reserved.

\section{Introduction}

Collagen is the most abundant protein in the body of mammals (approximately $30 \%$ of all proteins) and is a major structural component in the extracellular matrix (ECM) [4]. There are different diseases associated with excess collagen. The overproduction of

* Corresponding authors at: Departamento de Química en Ciencias Farmaceúticas, Facultad de Farmacia, Universidad Complutense de Madrid, 28040 Madrid, Spain.

E-mail addresses: abaezaga@ucm.es (A. Baeza),vallet@ucm.es (M. Vallet-Regí). collagen is due to fibroblast hyperproliferation or ECM remodeling imbalance, among other reasons [5]. The accumulation of this excess collagen within a tissue can lead to fibrosis, which compromises the tissue function, thereby resulting in physiological disorders and organ malfunction [6,7]. Fibrosis occurs in multiple conditions. For example, in superficial fibromatosis such as Dupuytren's contracture, which consists in formation of a fibrotic cord in the hand, thus resulting in hand dysfunction. In Peyronie's disease, penile involvement includes penile deviation, which makes sexual relationships difficult or even impossible. Keloids 
or hypertrophic scars are consequences of abnormal scarring. Some autoimmune diseases such as scleroderma characteristically present fibrotic/sclerous plaques on localized areas (morphea), generalized on the skin or even systemic (e.g., pulmonary involvement with lung fibrosis, resulting in high mortality rate).

Fibromatosis has been traditionally treated by surgery to remove the collagen deposition [6,8]. Unfortunately, surgical procedures often carry important complications, thereby producing serious adverse effects in many cases. In addition, the invasive nature of this type of intervention avoids the general application of surgical treatments, especially in fragile patients owing to a long postoperative recovery period [9]. The high prevalence of associated morbidity in surgical treatments has led to the development of other types of treatments such as radiotherapy, oral administration of vitamin E (tocopherol), or injection of corticosteroids, among others. However, these nonsurgical treatments have not demonstrated significant improvement in many cases $[4,10,11]$.

The administration of injections of collagenase Clostridium histolyticum $(\mathrm{CCH})$ has emerged as a promising nonsurgical alternative for the treatment of fibrotic diseases because it is minimally invasive and cost-effective as well as presents lower complications and adverse side effects $[7,9,12]$. CCH is a matrix metalloproteinase mixture of two synergistic microbial collagenases, AUX-I and AUXII, which act in combination and cleave the peptide bond of the collagen fibers between repeated sequences of Glycine-Proline-X (X being hydroxyproline or proline in the most effective cases) [4]. Collagenase is capable of lysing type 1 and type 3 collagens, which are the most abundant collagen types in fibrotic diseases. This enzyme shows a high specificity for collagen fibrils, whereas it does not produce any remarkable alteration in elastic fibers, vascular smooth muscle, and axonal myelin sheaths [10]. Thus, this enzyme has been tested in the treatment of a wide number of pathologies that course with an abnormal high regulation of formation and/or accumulation of collagen such as intervertebral disc herniation, [13] vitrectomy, [14] burns, [15] wound healing process, keloid, [1,16] and even in the treatment of solid tumors [17]. In the last application, the intratumoral injection of collagenase previous to the treatment with chemotherapeutic agents, both conventional drugs or nanoparticulated agents, allows a more homogeneous distribution of these therapeutic compounds along the tumoral tissue as a consequence of an improved diffusion of the molecules within a less dense ECM [18].

Owing to its probed effectiveness in some of these pathologies, $\mathrm{CCH}$ has been approved as an enzymatic treatment by the Food and Drug Administration in the USA in 2 February 2010 and by the European Agency for the Evaluation of Medicinal Products (EMEA) in January 2011, and it has been marketed under the name Xiaflex (Endo Pharmaceuticals, Inc) for the treatment of Dupuytren's contracture, a palmar fibromatosis [4]; in December 2013, it has been approved for the treatment of Peyronie's disease, a fibrosis that affects the tunica albuginea of the penis $[2,19]$. Label recommendations for the treatment of Dupuytren's contracture include up to three injections followed by finger extension $24-48 \mathrm{~h}$ after injection, which is usually a painful procedure. However, majority of the cases need only one injection on the fibrotic lesion [3]. Peyronie's disease requires up to eight collagenase injections followed by penile remodeling [20]. The need to apply repeated administration could be due to the low physico-chemical stability of collagenase, which suffers rapid proteolysis when it is exposed to the proteases present in tissues and also to relatively rapid denaturalization by oxidation or hydrolysis, among others. Thus, at physiological $\mathrm{pH}$, collagenase in solution practically lost all its activity after $24 \mathrm{~h}$, which indicates the labile nature of this macromolecule. Owing to the short half-life of collagenase, enzymatic activity is rapidly lost and efficacy is limited. Repeated injections followed by finger extension or penile remodeling are time consuming and very painful for patients as well as increase the risk of adverse events. For example, corporal ruptures of the penis have been reported to occur relatively frequent five days after the second injection of each cycle [21].

In view of these data, it is reasonable to think that injecting collagenase with long half-life could increase its efficacy, reduce the number of injections, spare working time of health professionals, and decrease the apparition of adverse events. Several alternatives have been evaluated to prolong the circulation time of these proteins, such as their transport immobilized on the surface of nanocarriers [22] or their encapsulation within polymeric shells [17]. However, these types of alternatives do not show a sustained release of the enzyme for a long time. This study presents a novel polymeric nanocapsule that contains collagenase encapsulated inside a matrix and is capable of releasing the enzyme with a controlled kinetics, thereby achieving a prolonged and sustained effect over time. Moreover, these nanocapsules protect the encapsulated collagenase against external aggressions, thus maintaining its catalytic activity for a long time. The efficacy of these nanocapsules has been tested using a mouse model of localized dermal fibrosis (scleroderma) induced by repeated dermal injection of bleomycin; these nanocapsules demonstrated a significant reduction in the fibrotic lesion compared to the conventional administration of free collagenase. These results could pave the way for the clinical use of these nanocapsules to treat localized fibrotic diseases. As an additional advantage, the highly versatile nature of the presented methodology can be easily applied for the encapsulation of different proteins, thus providing interesting alternatives of proteinbased diseases.

\section{Experimental section}

\subsection{Materials}

Collagenase Type I (Life Technologies); Acrylamide (AA) (Fluka); 2-Aminoethyl methacrylate hydrochloride (Am) (Sigma-Aldrich); Ethylene glycol dimethacrylate (EG) (Sigma-Aldrich); N,N'-Methy lenebis(acrylamide) (MBA) (Sigma-Aldrich); Ammonium persulfate (AP) (Sigma-Aldrich); N,N,N',N'-Tetramethylethylenediamine (TMDA) (Sigma-Aldrich); Amicon ${ }^{\circledR}$ Ultra-2 mL Centrifugal Filters Ultracel $^{\circledR} 10 \mathrm{~K}$ (Millipore); EnChek ${ }^{\circledR}$ Gelatinase/Collagenase Assay Kit (Life Technologies); 10X phosphate-buffered saline (PBS), buffer $\mathrm{pH}=7.4$ (Ambion).

\subsection{Instrumentation section}

The hydrodynamic size of protein capsules was measured by means of a Zetasizer Nano ZS (Malvern Instruments) equipped with a 633-nm "red" laser. Analysis using transmission electron microscopy (TEM) was carried out with a JEOL TEM 3000 instrument operated at $300 \mathrm{kV}$, equipped with a charge-coupled device (CCD) camera. Sample preparation was performed by dispersing these protein capsules in distilled water and subsequent deposition onto carbon-coated copper grids. A solution of $1 \%$ phosphotungstic acid (PTA), pH 7.0, was employed as the staining agent to visualize the protein capsules. Fluorescence was measured using Synergy 4; power supply for Biotek Laboratory Instrument was 100-240 VAC, $50 / 60 \mathrm{~Hz}$, and $250 \mathrm{~W}$.

\subsection{Synthesis of collagenase nanocapsules}

First, the reaction buffer $\mathrm{NaHCO}_{3}(0.01 \mathrm{M}, \mathrm{pH} 8.5)$ was deoxygenated by freeze-vacuum- $\mathrm{N}_{2}$ cycles. Then, collagenase $(3.1 \times 10$ ${ }_{-5} \mathrm{mmol}$ ) was dissolved in $1 \mathrm{~mL}$ of deoxygenated buffer. AA, Am, EG, and MBA, the proportions of which are required in each case, 
were dissolved in $1 \mathrm{~mL}$ of deoxygenated buffer in a vial, and the solution containing monomers was added to the protein solution. This mixture was stirred at $300 \mathrm{rpm}$ for $10 \mathrm{~min}$ under nitrogen atmosphere at room temperature. Further, $0.013 \mathrm{mmol}$ of AP and $0.02 \mathrm{mmol}$ of TMDA dissolved in $1 \mathrm{~mL}$ of the deoxygenated buffer were added to the mixture. This solution was stirred at $300 \mathrm{rpm}$ for $90 \mathrm{~min}$ at room temperature under inert atmosphere. Furthermore, the encapsulated enzyme was purified by centrifugal separation with 10-KDa cut-off filters (AMICON Ultra-2 mL $10 \mathrm{KDa}$ ) and washed three times with $\mathrm{NaHCO}_{3}$ buffer $(0.01 \mathrm{M}, \mathrm{pH} 8.5)$. The capsules of collagenase were preserved at $4{ }^{\circ} \mathrm{C}$.

\subsection{Enzymatic activity assay}

The samples were incubated using the same collagenase concentration (measured by absorbance at $280 \mathrm{~nm}$ ) in PBS with physiological $\mathrm{pH}=7.4$ and at $37^{\circ} \mathrm{C}$ under stirring. The encapsulation process does not affect the absorbance because the monomers and polymers created do not have absorbance at this wavelength. At certain times, an aliquot of this solution was removed and its enzymatic activity was evaluated using the commercial kit (EnzChekGelatinase/Collagenase Assay Kit). The kit provides a fluorimetric method, and its protocol consists in mixing the collagenase sample with commercial buffer, which contains calcium and fluorescent-labeled gelatin. The fluorescence signal is quenched until gelatin digestion. Then, the enzymatic activity of the collagenase sample was determined by fluorescence release.

\subsection{Hydrolysis assay}

Collagenase nanocapsules were incubated in PBS with physiological $\mathrm{pH}=7.4$ and at $37{ }^{\circ} \mathrm{C}$ under stirring. At certain times, an aliquot was removed from the solution and stained with $1 \%$ phosphotungstic acid; these nanocapsules were then observed by TEM.

\subsection{Mouse model of scleroderma}

Female 6-week-old $\mathrm{C} 3 \mathrm{H} / \mathrm{HeNH}$ se mice were purchased from Envigo (Valencia, Spain). Dermal fibrosis was induced by subcutaneous injections of $100 \mu \mathrm{g}$ of bleomycin $\left(1 \mathrm{mg} \cdot \mathrm{ml}^{-1}\right.$; Mylan Pharmaceuticals, Barcelona, Spain) or $0.9 \%$ saline control into the shaved skin at the back every day for 4 weeks as previously described [23]. To analyze the effect of collagenase administration in this model after fibrosis induction, 10 mice in each group were administered with $300 \mu \mathrm{g}$ of collagenase (Life Technologies), either as a single subcutaneous injection of free collagenase or encapsulated nanocapsules, in the bleomycin-injected skin area and maintained for 10 days. To ensure that the amount of injected free and encapsulated collagenase is the same, the total protein content was determined by absorbance at $280 \mathrm{~nm}$. The study was approved by Animal Care and Use Committee of Hospital 12 de Octubre with protocol reference number PROEX 407/15, and this was carried out in accordance with the institutional guidelines.

The treated skin was harvested, and paraffin was embedded for histological evaluation of the collagen dermal area by using the Masson's trichrome staining kit (Sigma-Aldrich, St. Louis, USA). Masson-stained full-thickness skin sections were photographed and digitalized using an AxioCam ERc 5S camera and ZEN lite 2012 software (Zeiss, Jena, Germany). The blue-stained collagen fractional area was quantified using Image software (http://rsb. info.nih.gov/ij). The collagen area of each individual mouse was calculated as the mean area of several (three to five) sections of the central part of the excised skin piece.

\subsection{Statistical analyses}

Data were analyzed using Prism software (GraphPad Software, San Diego, CA, USA). Results are expressed as mean \pm standard deviation (SD), and quantitative data were analyzed by the Mann-Whitney $U$ test. P values $<0.05$ were considered significant.

\section{Results and discussion}

In this work, a protective polymer capsule is designed around the collagenase whose function is to release the encapsulated enzyme in a controlled sustained manner for a long period of time. In this way, the polymeric nanocapsule acts as collagenase reservoir that presents a slow and tunable kinetic release capable of providing a prolonged effect, thus avoiding the need for important and repeat doses.

Protein nanocapsules have been widely studied to obtain a controllable delivery system that also protects the cargo at the same time. In this sense, the development of a protein carrier based in its encapsulation within the polymeric mesh has received increasing attention in the last few years [24]. This type of system has demonstrated its capacity to trigger the protein release in response to different stimuli, for example, enzymes present in the target site, according to local environment or specific cellular events [25]. In addition, these protein nanocapsules have been designed to achieve a sequential release of multiple proteins, so that the release of the first protein triggers the release of the rest in a tandem sequence [26]. As an added value, polymeric nanocapsules act as a protective coating for the encapsulated enzyme against external stresses that can compromise its structure and, therefore, its catalytic activity [17].

Thus, with the objective to obtain a collagenase nanocapsule that provides a sustained release, a polymeric mesh was designed to coat the protein. Our nanocapsules have been synthesized by free radical polymerization of two types of monomers, i.e., a neutral monomer, AA, and Am. AA is widely used as a structural monomer, whereas Am presents the capacity to adsorb around negative proteins by electrostatic affinity owing to its positive charge in the aqueous phase. Thus, the enrichment of monomers around the proteins is favored by electrostatic bonding. Additionally, once the nanocapsule is formed, the presence of these amino groups on the surface avoids capsule aggregation by repulsion charge, thereby enhancing the colloidal stability of the system. Finally, EG was chosen as a degradable crosslinker to form the polymeric shell [27].

First, the monomers and protein are allowed to come in contact in an aqueous solution free of oxygen. The protein and monomer mixture was kept under stirring for $10 \mathrm{~min}$ to allow the adsorption of the monomers on the negative surface of protein through intermolecular interactions. Then the protein surface could be enriched with monomers in such a way that the polymerization occurs around the protein. Oxygen, which can terminate the radical polymerization process, is removed from the buffer reaction previous to the addition of radical initiators. This first stage is followed by a polymerization step. The polymerization is initiated by the addition of AP in the presence of TMDA, and this forms free radicals of oxygen in the aqueous solution at room temperature through a basic catalysis mechanism. AP is the initiator of free radical generation and this is catalyzed by TMDA, which promotes the decomposition of AP into free radicals, thereby decreasing the activation energy of polymerization [28]. In this way, the addition of TMDA allows the free radical polymerization process to occur at room temperature. The free radical process leads to polymerization of acryloyl groups of monomers and crosslinker, thus yielding a polymeric mesh that coats the protein (Scheme 1). The mixture 

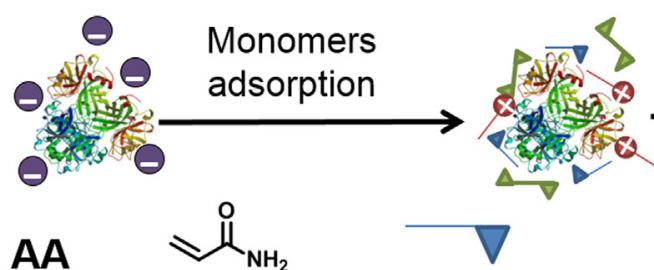

\section{Free Radical Polymerization \\ PA/TMDA}<smiles>C=C(C)C(=O)OCCN</smiles>
EG<smiles>C=CC(=O)OCCOC(=O)C=C</smiles><smiles>C1CCCC1</smiles>

\section{Polymerization Process}

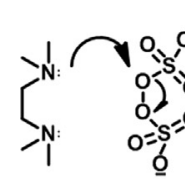

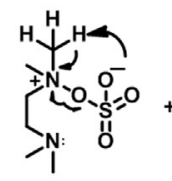<smiles>O=S(=O)([O-])O</smiles>

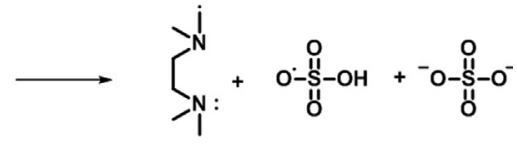<smiles>[R]CC=CC(=O)OCCOC(=O)C=CC(=O)OCCN</smiles>

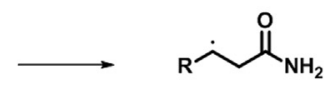<smiles>C1CCCCC1</smiles><smiles>[R]CC(C)C(=O)OCCN</smiles><smiles>CCCC(C)C(=O)CCOC(=O)C(C)C</smiles>

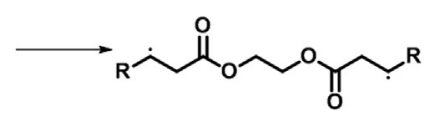

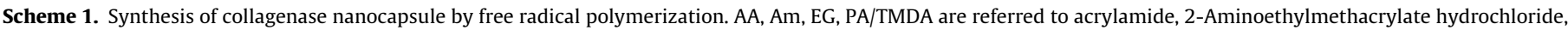
ethylene glycol dimethacrylate, ammonium persulfate/ $N, N, N^{\prime}, N^{\prime}$, tetramethylenediamine respectively.

was kept at room temperature for $90 \mathrm{~min}$, and collagenase nanocapsules were isolated by centrifugal separation with 10 KDa cut-off filters to remove the excess monomer. Collagenase acts on large substrates (collagen fibers), and therefore, it is necessary to consider its release to facilitate its catalytic activity. To provide a controlled release mechanism, EG was chosen as a pH-responsive crosslinker. Thus, the obtained nanocapsules would be degradable by hydrolysis. The collagenase release mechanism is shown in Scheme 2. In the aqueous medium, water molecules act as nucleophiles that attack the carbonyl group of the crosslinker, which results in the hydrolysis of the ester bonds. This crosslinker degradation is catalyzed in acidic environments because of an interaction of protons with the oxygen atom of the carbonyl group, thus increasing the polarity of the covalent bond and favoring the nucleophilic attack by water.

\subsection{Optimization of collagenase synthesis for sustained release}

\subsubsection{Previous work}

Our research group recently reported the encapsulation of collagenase inside degradable polymeric nanocapsules using a protein-to-monomer ratio of 1:2025 and AA-to-Am-to-EG monomer ratio of 7:6:2 [17]. These conditions favored the nanocapsules to release the encapsulated enzyme in $<12 \mathrm{~h}$ under physiological conditions. This presumes a high improvement in the stability of the enzyme, and this relatively rapid release is suitable for certain clinical applications such as in the case of fibrotic disease, a longer release time is required as mentioned.

\subsubsection{Strategies to achieve a sustained and prolonged release}

Based on the previous work, different strategies have been performed to achieve a prolonged and sustained release. In this way, it is hoped that the collagenase nanocapsules can act a depot of the proteolytic enzyme and improve the temporal window by enhancing its therapeutic effect. Two strategies were developed to achieve this goal; first, the degradable crosslinker ratio was reduced to reduce the hydrolyzable attack points in the polymeric mesh, and second, obtaining collagenase nanocapsules with sustained release was addressed from another point of view, that is, the addition of an additional nondegradable crosslinker; in this way, the polymeric mesh would become more robust and the collagenase release rate would decrease.

3.1.2.1. Decrease in the proportion of degradable crosslinker. To decrease the hydrolysis rate of the collagenase nanocapsules, the amount of degradable crosslinker was reduced to half with regard to a previous work [17]. The protein-to-monomer ratio was maintained constant because a significant alteration in this ratio can lead to poor protein encapsulation. The capacity to provide a sustained enzymatic activity of these nanocapsules for a long time was evaluated by incubating the nanocapsules in an aqueous solution at physiological $\mathrm{pH}$ and temperature under orbitalic soft stir- 


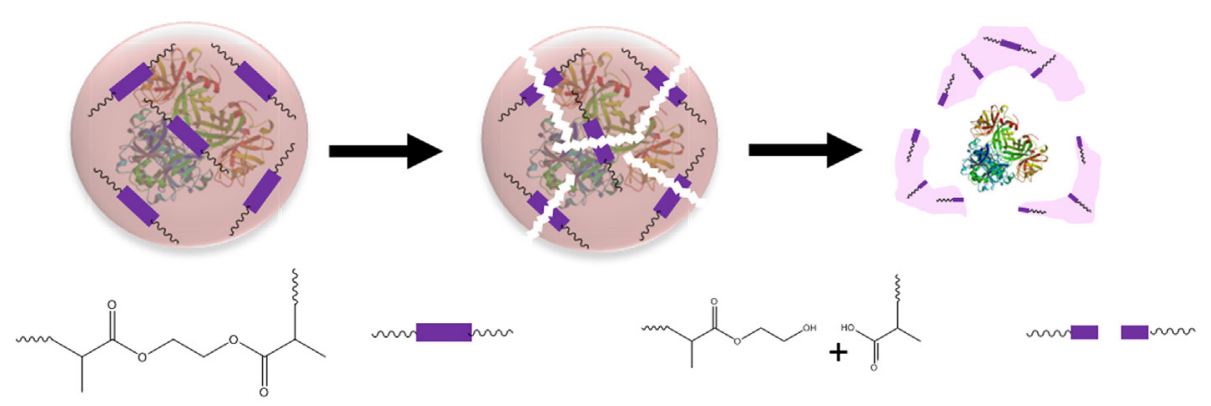

Degradation in Acidic Environment

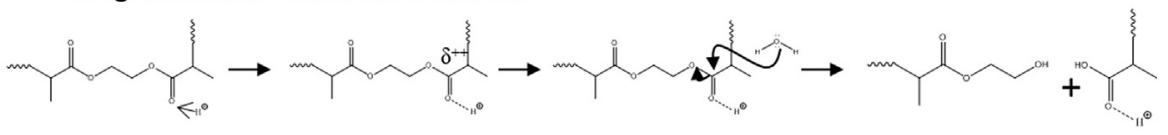

Degradation in Neutral Environment

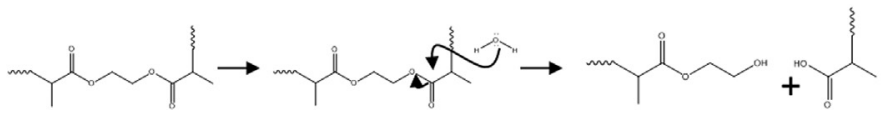

Scheme 2. Degradation mechanism of the collagense nanocapsules.

ring. Then, at certain times, an aliquot of this solution was removed, and the enzymatic activity was measured using a commercial kit protocol. The nanocapsules retain more than $50 \%$ of the catalytic capacity initially for 2 days, which is a significant improvement compared to the free enzyme, which lost more than $80 \%$ of its capacity after 1 day. The encapsulation efficacy is also dependent on the capacity of the monomers to get adsorbed on the protein surface, and this capacity is related to the intermolec-

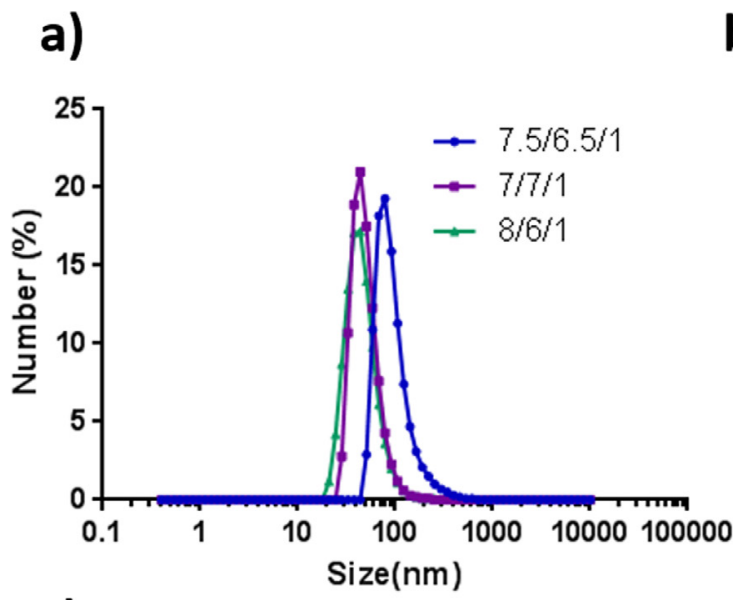

b)

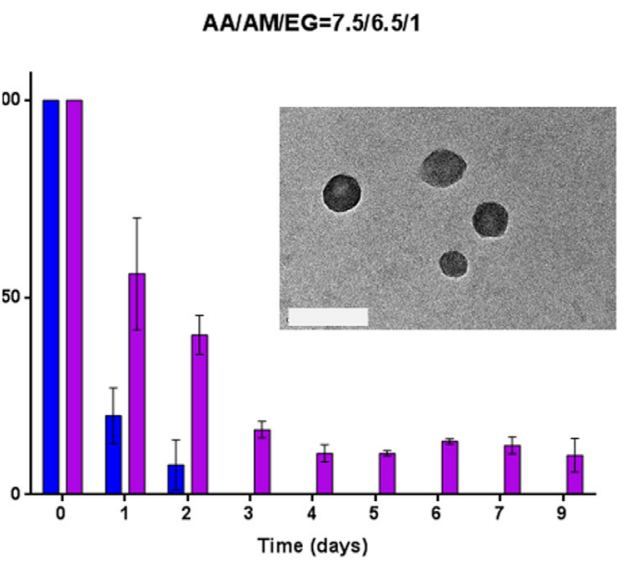

c)

AA/AMEG $=7 / 7 / 1$

d)
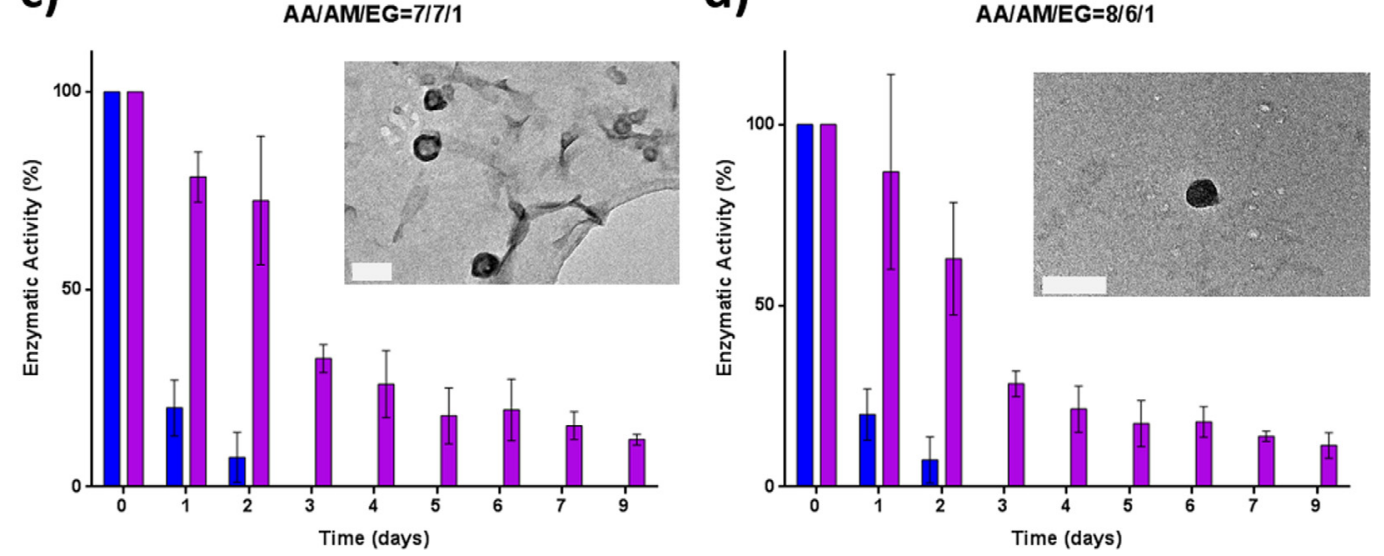

Fig. 1. (a) Study of Dynamic Light Scaterring (DLS) of collagenase nanocapsules with different AA/Am ratios. Study of stability of collagenase nanocapusules with different ratios AA/Am (purple). Micrograph by transmission Electron Microscopy (b) $A A / A m / E G=7.5 / 6.5 / 1$, (c) $A A / A m / E G=7 / 7 / 1$ and (d) $A A / A m / E G=8 / 6 / 1$ (white scale bar correspond to $100 \mathrm{~nm})$. Study of free collagenase was represented in blue. 
ular interactions between both systems. To evaluate whether the AA-to-Am monomer ratio could play a role in this process, two different monomer ratios were also evaluated, i.e., AA-to-Am-toEG ratios of 7:7:1 and 8:6:1, respectively. Neither of these cases provided a significant improvement in the catalytic activity during time, thus losing more than $50 \%$ of their capacity after 2 days, similar to the results of the previous work (Fig. 1).

The presence of a crosslinker is strictly needed to obtain a dense and protective mesh around the protein. Although a high decrease in the crosslinker ratio could be an option to extend the release time, it could result in an inappropriate capsule formation. For this reason, the possibility to reduce its amount even more was discarded and the other strategy was addressed.

3.1.2.2. Introduction of a nondegradable crosslinker. Instead of reducing the crosslinker ratio, a novel strategy that consists in the introduction of an additional nondegradable crosslinker, MBA, was employed. The introduction of this crosslinker should not alter the nanocapsule formation because the crosslinker-tomonomer ratio was maintained and could increase the degradation time of the nanocapsule, thus allowing a sustained release for a longer time. Although the addition of a nondegradable crosslinker can result in incomplete collagenase release, this strategy decreases the hydrolyzable points in the polymeric mesh without altering the crosslinker ratio. Thus, with the protein-to-monomer ratio as 1:2025 and AA-to-Am-to-crosslinker ratio as 7:6:2, which provided a good encapsulation capacity, different ratios of both degradable and nondegradable crosslinkers were studied. The addition of the new crosslinker did not alter the size and morphology of the nanocapsules in all cases. The results indicate that employing a 1:1 ratio of degradable and nondegradable crosslinkers enables the system to maintain the enzymatic activity for a really long time. This sample shows a sustained release of around $50 \%$ of its initial activity lasting for 10 days and maintaining $15 \%$ of its activity for 12 days (Fig. 2). Surprisingly, for unknown reasons, when different ratios of the two crosslinkers are employed, the capacity to maintain the catalytic activity reduces to values even lower than those of the previous systems that carry only the degradable crosslinker, i.e., when the nondegradable crosslinker is employed both in a higher ratio and in a lower ratio. In any case, the system that employs a 1:1 ratio exhibits excellent properties. The hydrolysis of this sample was observed in the micrographs obtained by TEM (Fig. 3). It can be observed that the collagenase nanocapsules progressively lost their integrity as is expected because they are degradable nanocapsules. Owing to their sustained release, a sample with an EG-to-MBA ratio as 1:1 was chosen for in vivo evaluation in comparison with the current treatment.

Therefore, two strategies were evaluated to achieve a sustained and prolonged release of collagenase. Both approaches demonstrated an increase in the collagenase release time with regard to our previous work. In the first study, a decrease in degradable crosslinker ratio in the synthesis of collagenase nanocapsules was evaluated. This strategy was based on the idea that decreasing the degradable crosslinker ratio led to a reduction in the hydrolyzable attack points. Although the stability of the collagenase nanocapsules was higher than that of the free enzyme, the results were not satisfactory because the collagenase release was not sustained, losing almost completely the enzymatic activity after 3 days. The idea to continue reducing the crosslinker ratio was dis-

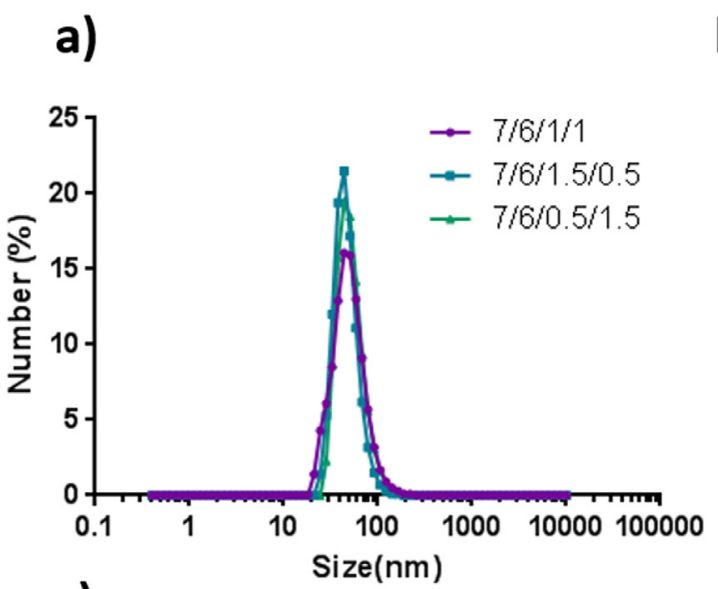

b)

AA/AM/EG/MBA $=7 / 6 / 1 / 1$
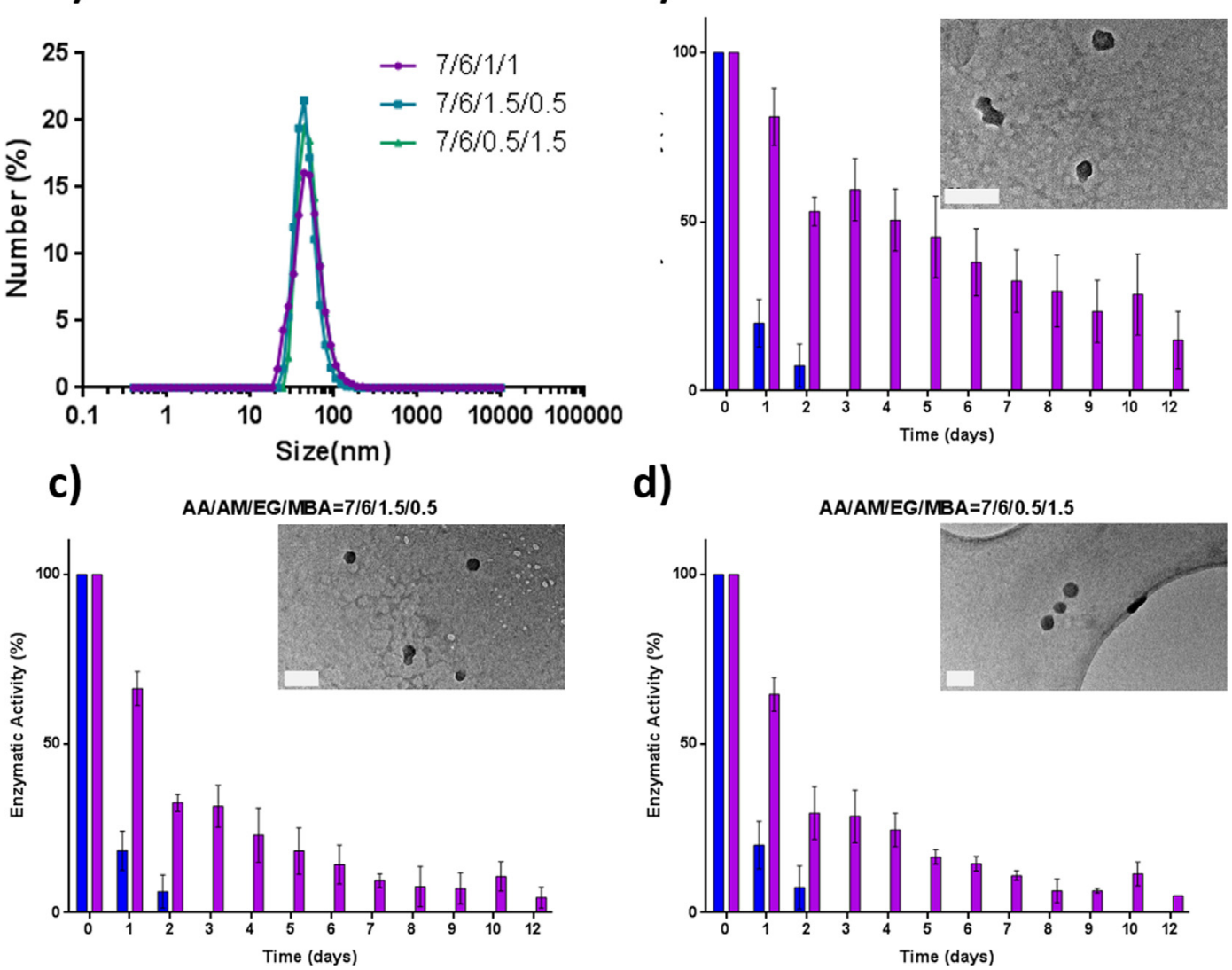

d)

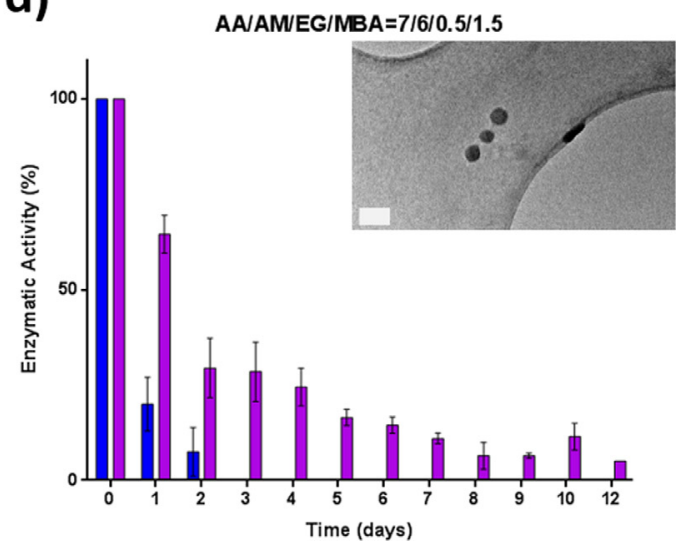

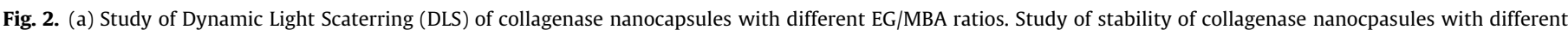

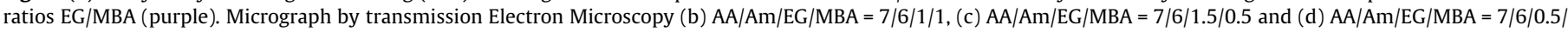
1.5 (white scale bars correspond to $100 \mathrm{~nm}$ ). Study of free collagenase was represented in blue. 


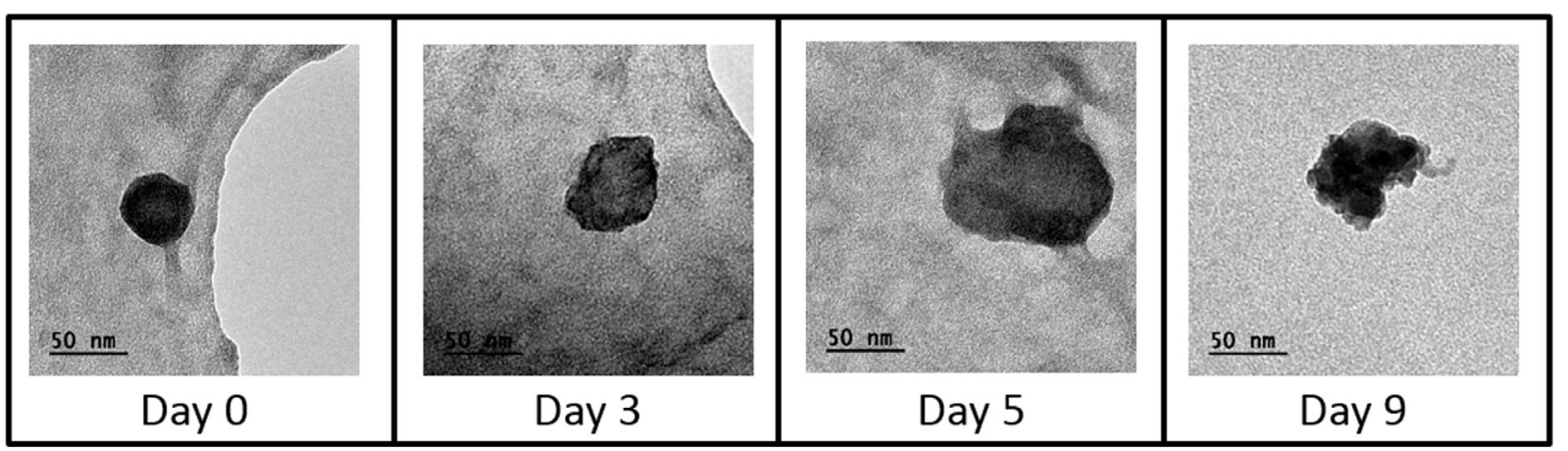

Fig. 3. TEM micrographs of collagenase nanocapsules incubated at physiological conditions at different times.

carded because the presence of crosslinker is necessary to obtain a robust polymeric coating. Thus, the problem was addressed from the other point of view, by the introduction of an additional nondegradable crosslinker. Despite the fact that the addition of a nondegradable crosslinker presented a potential liability regarding the possibility to lead to an incomplete collagenase release, it decreased the hydrolysis-sensitive points in the nanocapsules without altering the crosslinker ratio. This approach yielded the nanocapsules with excellent properties for the treatment of fibrotic lesions because they exhibited a sustained and prolonged collagenase release for up to 10 days. Once the synthetic conditions for the formation of collagenase nanocapsules with desired properties are optimized, the suitability to treat fibrotic lesions with this nanodevice was evaluated in a mice model as proof of concept in comparison with the conventional free enzyme administration.

\subsection{In vivo cytotoxicity test of collagenase nanocapsules}

To check the biocompatibility of the collagenases nanocapsules in vivo, a fixed amount of nanocapsules or free collagenase was injected subcutaneously into the shaved skin at the back of healthy mice. After 10 days, the animals were sacrificed and histological analysis of collagenase-injected skin showed a normal structure of all skin layers and absence of inflammatory cell infiltration (Fig. 4). Dermal collagen area and structure did not show changes compared to the normal (uninjected) skin (Normal skin $v s$. free collagenase, $\mathrm{p}=0.7$; normal skin $v$ s. nanocapsules, $\mathrm{p}=0.99$; and free collagenase $v s$. nanocapsules, $\mathrm{p}=0.628$ ).

\subsection{In vivo evaluation of the efficacy of collagenase nanocapsules}

To study the efficacy of collagenase nanocapsules on bleomycin-induced skin fibrosis, we evaluated changes in the fibrotic area induced by bleomycin for 4 weeks, after injection of either free collagenase or collagenase nanocapsules. We found a marked increase in the dermal collagen area of the dermis after bleomycin injection compared to control (saline-injected) group (Fig. 5).

The fibrotic area, evaluated as the fractional collagen-stained area (blue-stained area) of the dermis, was significantly reduced
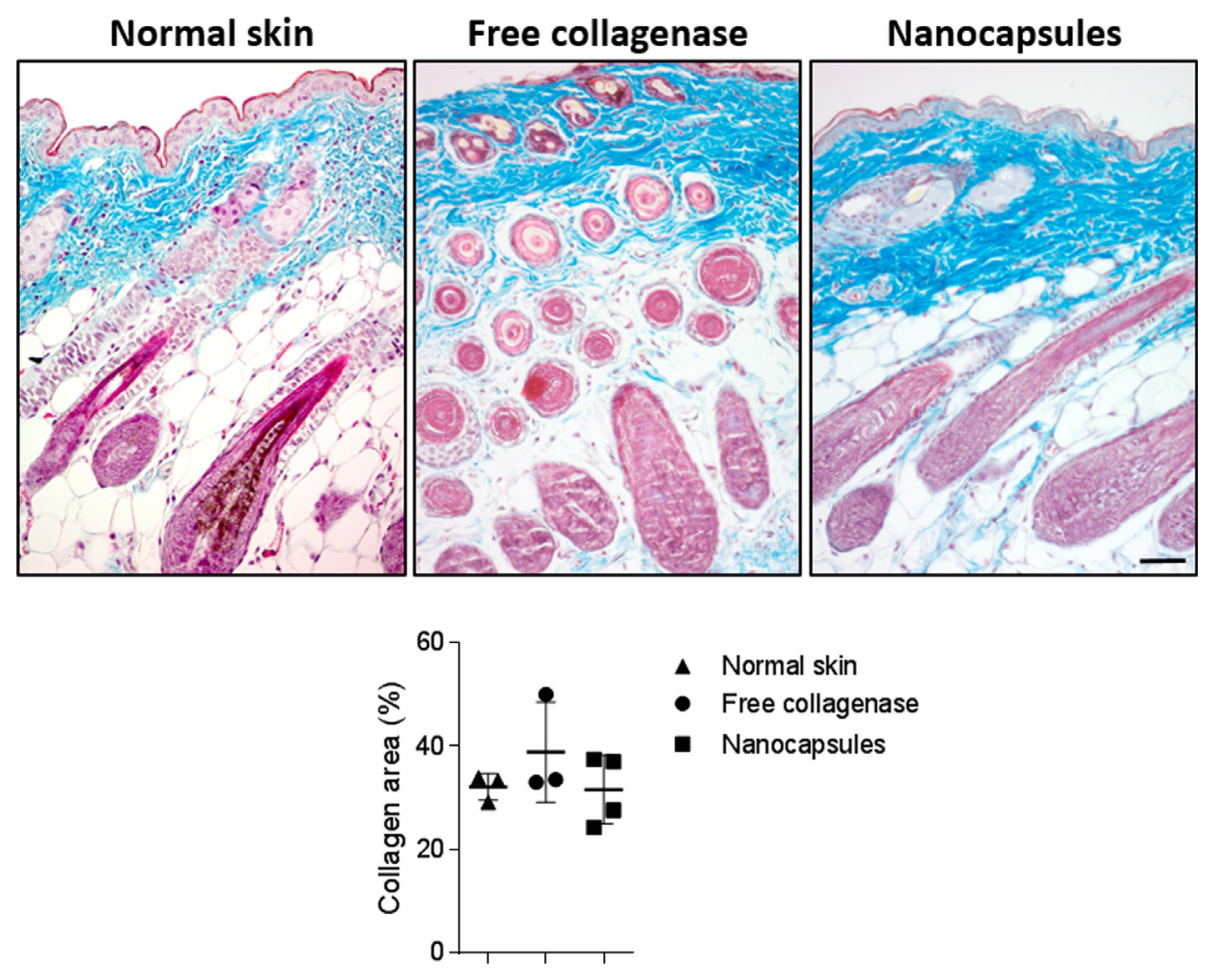

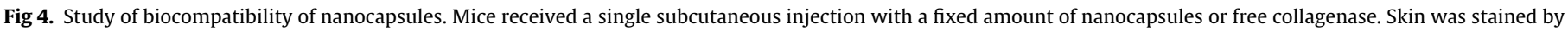
Masson's trichrome (collagen fibers are stained in blue). Data are representative of one experiment with three-four mice per group. Bar $50 \mu \mathrm{m}$. 


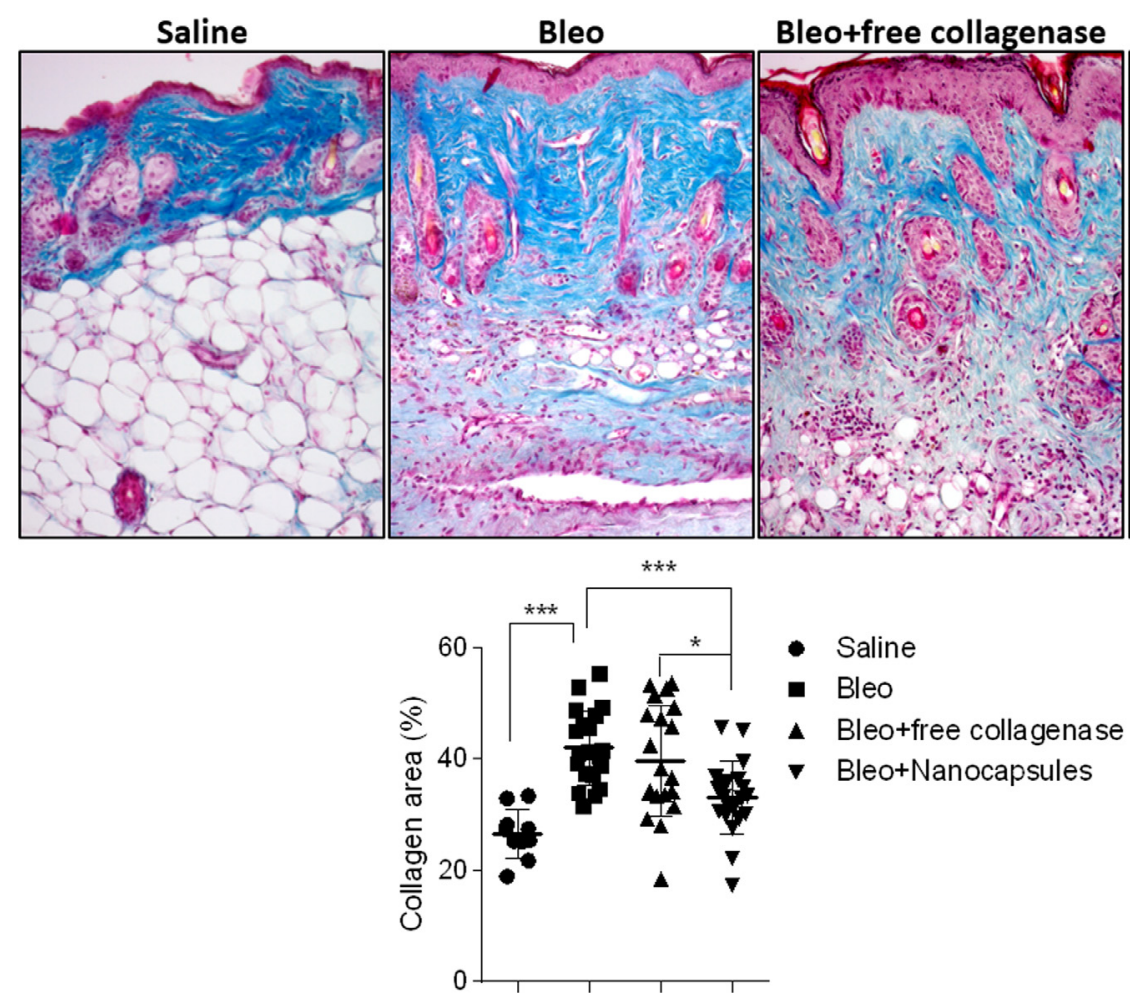

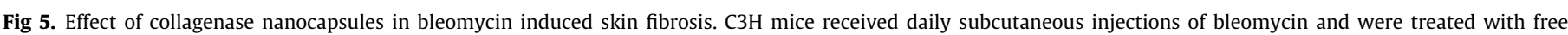

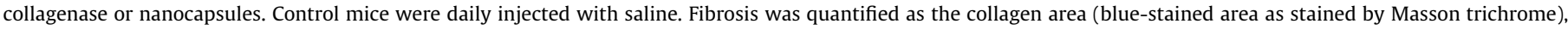
expressed as a fraction of the total skin area. Data are representative of two independent experiments with 10 mice per group. Bar $50 \mu \mathrm{m}$.

bleomycin-injected, collagenase nanocapsule-treated mice compared to those in bleomycin-injected mice $(p<0.0001)$ and bleomycin-injected, free collagenase-treated mice $(p=0.026)$. There were no significant differences between bleomycininjected, free collagenase-treated and bleomycin-injected groups $(p=0.457$; Fig. 5). Therefore, administration of a single dose of encapsulated collagenase nanocapsules significantly decreased the collagen area, whereas the same dose of collagenase without encapsulation (free collagenase) was insufficient to reduce the collagen area.

The fact that a single injection of collagenase nanocapsules results in a significant decrease in the collagen area with regard to free enzyme could presume an important advance the treatment for fibrotic lesions. In the future, preclinical studies should be organized to conduct clinical trials in humans.

\section{Conclusion}

This study presents a novel collagenase nanocapsule capable of a sustained release and delivery of the proteolytic enzyme. These collagenase nanocapsules were designed to protect the activity of the collagenase enzyme and release with controllable kinetics for long and modular periods of time. These polymeric nanocapsules presume a delivery system of collagenase capable of releasing the enzyme under physiological conditions for 10 days.

This type of release is highly beneficial in enzymatic treatments because a prolonged effect of the required enzyme over time is obtained, thus allowing the use of lower doses and/or reducing the number of injections necessary to achieve acceptable results. The high stability conferred by this coating composition coupled with the sustained release profile results in a promising advance in the stabilization of the enzyme and makes it possible to improve the current clinical treatment. It has been observed in fibrotic mod- els that the encapsulated collagenase showed a high efficacy of degradation compared to that of free enzyme, which is the actual treatment. The application of this strategy would pave way for the proteins to get delivered in a more sustained release, which would provide an important advance in their clinical applications.

\section{Acknowledgments}

The authors acknowledge the financial support provided by European Research Council (Advanced Grant VERDI; ERC-2015AdG Proposal No. 694160) and the project MAT2015-64831-R. In addition, this work was supported by grants from the Instituto de Salud Carlos III (Ministerio de Economía y Competitividad, Spain) PI 12/439 and RIER (Red de Investigación en Inflamación y Enfermedades Reumáticas) to AU and JLP as well as cofinanced by FEDER (European Union).

\section{References}

[1] Y.-S.C. Bae-Harboe, J.E. Harboe-Schmidt, E. Graber, B.A. Gilchrest, Collagenase Followed by Compression for the Treatment of Earlobe Keloids, Dermatologic Surg. 40 (2014) 519-524, https://doi.org/10.1111/dsu.12465.

[2] J. Akerman, J.R. Kovac, Treatment of Peyronie's disease via preoperative intralesional collagenase clostridium histolyticum followed by placement of an inflatable penile prosthesis: the new standard of care?, Transl Androl. Urol. 6 (2017) S822-S823, https://doi.org/10.21037/tau.2017.11.04.

[3] D.J. Warwick, D. Graham, P. Worsley, New insights into the immediate outcome of collagenase injections for Dupuytren's contracture, J. Hand Surg. European 41 (2016) 583-588. doi:10.1177/1753193415600670.

[4] A. Bayat, A. Thomas, The emerging role of Clostridium histolyticum collagenase in the treatment of Dupuytren disease, Ther. Clin. Risk Manag. 6 (2010) 557572, https://doi.org/10.2147/TCRM.S8591.

[5] T.R. Cox, J.T. Erler, Remodeling and homeostasis of the extracellular matrix: implications for fibrotic diseases and cancer, Dis. Model. Mech. 4 (2011) $165-$ 178, https://doi.org/10.1242/dmm.004077.

[6] N.W. Bulstrode, B. Jemec, P.J. Smith, The complications of Dupuytren's contracture surgery, J. Hand Surg. Am. 30 (2005) 1021-1025, https://doi.org/ 10.1016/j.jhsa.2005.05.008. 
[7] M. Gelbard, I. Goldstein, W.J.G. Hellstrom, C.G. McMahon, T. Smith, J. Tursi, N. Jones, G.J. Kaufman, C.C. Carson, Clinical efficacy, safety and tolerability of collagenase clostridium histolyticum for the treatment of peyronie disease in 2 large double-blind, randomized, placebo controlled phase 3 studies, J. Urol. 190 (2013) 199-207, https://doi.org/10.1016/j.juro.2013.01.087.

[8] S.S. Desai, V.R. Hentz, N. Orthopedic, N. Beach, R.A.C. Hand, U. Limb, The Treatment of Dupuytren Disease, YJHSU. 36 (2011) 936-942, https://doi.org/ 10.1016/j.jhsa.2011.03.002.

[9] F. Smeraglia, A. Del Buono, N. Maffulli, Collagenase clostridium histolyticum in Dupuytren's contracture: a systematic review (2016) 1-10, https://doi.org/ $10.1093 / \mathrm{bmb} / \mathrm{ldw020}$.

[10] A. Gokce, J.C. Wang, M.K. Powers, W.J. Hellstrom, Current and emerging treatment options for Peyronie's disease, Res. Reports Urol. 5 (2013) 17-27, https://doi.org/10.2147/RRU.S24609.

[11] A.N. Bilgutay, A.W. Pastuszak, Peyronie's Disease: A Review of Etiology, Diagnosis, and Management, Curr. Sex. Heal. Rep. 7 (2015) 117-131, https:// doi.org/10.1007/s11930-015-0045-y.

[12] R. Arora, P. Kaiser, T.-J. Kastenberger, G. Schmiedle, S. Erhart, M. Gabl Injectable collagenase Clostridium histolyticum as a nonsurgical treatment fo Dupuytren?s disease, Oper. Orthop?die Und Traumatol. 28 (2016) 30-37. doi:10.1007/s00064-015-0434-4.

[13] D. Zhang, Y. Zhang, Z. Wang, X. Zhang, M. Sheng, Target radiofrequency combined with collagenase chemonucleolysis in the treatment of lumbar intervertebral disc herniation, Int. J. Clin. Exp. Med. 8 (2015) 526-532.

[14] C. Moorhead, D.S. Kirkpatrick, F. Kretzer, Collagenase With Proposed Adjunct to Vitrectomy (n.d). doi:10.1001/archopht.1980.01020040681018.

[15] N.E. Sharp, P. Aguayo, D.J. Marx, R.N.E.E. Polak, D.E. Rash, P.C. Shawn, D.S. Peter, D.J. Ostlie, D. Juang, Nursing Preference of Topical Silver Sulfadiazine Versus Collagenase Ointment for Treatment of Partial Thickness Burns, Children (2014) 253-257, https://doi.org/10.1097/JTN.0000000000000073.

[16] N. Kang, B. Sivakumar, R. Sanders, C. Nduka, D. Gault, Intra-lesional injections of collagenase are ineffective in the treatment of keloid and hypertrophic scars, J. Plast. Reconstr. Aesthetic Surg. 59 (2006) 693-699, https://doi.org/ 10.1016/j.bjps.2005.11.022.

[17] M.R. Villegas, A. Baeza, M. Vallet, Regí, Hybrid Collagenase Nanocapsules for Enhanced Nanocarrier Penetration in Tumoral Tissues, ACS Appl. Mater. Interfaces 7 (2015) 24075-24081, https://doi.org/10.1021/acsami. 5b07116.
[18] T.D. McKee, P. Grandi, W. Mok, G. Alexandrakis, N. Insin, J.P. Zimmer, M.G. Bawendi, Y. Boucher, X.O. Breakefield, R.K. Jain, Degradation of fibrillar collagen in a human melanoma xenograft improves the efficacy of an oncolytic herpes simplex virus vector, Cancer Res. 66 (2006) 2509-2513, https://doi.org/10.1158/0008-5472.CAN-05-2242.

[19] L.A. Levine, S.M. Larsen, Surgical Correction of Persistent Peyronie's Disease Following Collagenase Clostridium Histolyticum Treatment, J. Sex. Med. 12 (2015) 259-264, https://doi.org/10.1111/jsm.12721.

[20] Xiaflex (Collagenase Clostridium Histolyticum), Prescribing Information Malvern, PA: Auxilim Pharmaceuticals, 2010, pp. 1-42.

[21] S. Yan, T. Yap, S. Minhas, Collagenase clostridium histolyticum intralesional injections for the treatment of Peyronie's disease: a safety profile 6 (2017) 123-126, https://doi.org/10.21037/tau.2016.12.08.

[22] S.J. Kuhn, S.K. Finch, D.E. Hallahan, T.D. Giorgio, Proteolytic surface functionalization enhances in vitro magnetic nanoparticle mobility through extracellular matrix, Nano Lett. 6 (2006) 306-312, https://doi.org/10.1021/ nl052241g.

[23] B. Santiago, I. Gutierrez-Cañas, J. Dotor, G. Palao, J.J. Lasarte, J. Ruiz, J. Prieto, F. Borrás-Cuesta, J.L. Pablos, Topical application of a peptide inhibitor of transforming growth factor- $\beta 1$ ameliorates bleomycin-induced skin fibrosis, J. Invest. Dermatol. 125 (2005) 450-455, https://doi.org/10.1111/j.0022202X.2005.23859.X.

[24] Y. Lu, W. Sun, Z. Gu, Stimuli-responsive nanomaterials for therapeutic protein delivery, J. Control. Release 194 (2014) 1-19, https://doi.org/10.1016/j. jconrel.2014.08.015.

[25] J. Wen, S.M. Anderson, J. Du, M. Yan, J. Wang M. Shen, Y. Lu, T. Segura, Controlled protein delivery based on enzyme-responsive nanocapsules, Adv. Mater. 23 (2011) 4549-4553, https://doi.org/10.1002/adma.201101771.

[26] S. Zhu, L. Nih, S.T. Carmichael, Y. Lu, T. Segura, Enzyme-Responsive Delivery of Multiple Proteins with Spatiotemporal Control, Adv. Mater. 27 (2015) 36203625, https://doi.org/10.1002/adma.201500417.

[27] C. Liu, J. Wen, Y. Meng, K. Zhang, J. Zhu, Y. Ren, X. Qian, X. Yuan, Y. Lu, C. Kang, Efficient delivery of therapeutic miRNA nanocapsules for tumor suppression, Adv. Mater. 27 (2015) 292-297, https://doi.org/10.1002/adma.201403387.

[28] B. Strachota, L. Matějka, A. Zhigunov, R. Konefał, J.í Spěváček, J.í Dybal, R. Puffr, Poly(N-isopropylacrylamide)-clay based hydrogels controlled by the initiating conditions: evolution of structure and gel formation (n.d.). doi:10.1039/ c5sm01996f. 